\title{
CRISPR technology challenge facing the numerical integrity of whole human genome DNA
}

\begin{abstract}
Background: Global analysis of 3 human genomes of increasing levels of evolution (Neanderthal/Sapiens Build34/Sapiens hg38) reveals 2 levels of numerical constraints controlling, structuring and optimizing these genome's DNA sequences. A global constraint - called "HGO" for "Human Genome Optimum"-optimizes the genome at its global scale. The same operator applied to each of the 24 individual chromosomes reveals a hierarchical structure of these 24 chromosomes.
\end{abstract}

Results: Then analysing the single strand DNA CG/TA proportions at whole chromosomes and genome scale reveals strong fine-tuned numerical ratios evidencing the "closure" nature (Varela's autopoiesis theory) of whole human genome.

Keywords: human genome, CRISPR,; biomathematics, evolution autopoiesis, DNA
Volume I Issue 6 - 2017

Jean-Claude Perez

Retired interdisciplinary researcher (IBM), France

Correspondence: Jean-Claude Perez, Retired interdisciplinary researcher (IBM), 7 avenue de terre-rouge F33I27 Martignas Bordeaux metropole, France,

Email jeanclaudeperez2@gmail.com

Received: July 27, 2017 | Published: October 05, 2017

\section{Introduction}

Thanks to the CRISPR (Clustered regularly interspaced short palindromic repeats) technology, it is now possible to locally modify the genomes, and particularly the human genome. ${ }^{1}$ Almost simultaneously, the fractal and global structures of the human genome were demonstrated. ${ }^{2}$ In such a context, apart from ethical questions, can a local technology as powerful as CRISPR be applied, ignoring its possible effect on the possible global and long-range equilibria and balancing at the chromosome scale or even the entire genome scale? For more than 25 years, we have been looking for possible global, even numerical, structures that would organize DNA, genes, chromosomes and even whole genomes. ${ }^{3-9}$ We have already demonstrated a numerical structure at the scale of each human chromosome as well as on the whole genome. ${ }^{10-15} \operatorname{In}^{10}$ we have already highlighted this numerical value of 0.6909830056, the HGO (Human Genome Optimum) in this article: it controls the population of triplets codons analysing single stranded DNA sequence from the whole human genome.

\section{Materials and methods}

\section{Analysed whole human genomes}

We analyzed completely and systematically each of the 24 chromosomes of each of the following three reference genomes:

Neanderthal genome: (2014) ref ${ }^{16}$

http://www.nature.com/nature/journal/v505/n7481/full/ nature12886.html

Sapiens Build34 (2003) human reference genome $\operatorname{ref}^{17}$

http://www.nature.com/nature/journal/v431/n7011/full/ nature03001.html

Sapiens hg38 (2013) human reference genome ref ${ }^{18}$

https://www.ncbi.nlm.nih.gov/grc/human

Computing the HGO (Human Genome Optimum): let us now distinguish the two types of HGO that will be discussed

1/Theoretical HGO (tHGO)
tHGO $=(3-\mathrm{Phi}) \div 2=0.6909830056$, where Phi is the Golden Ratio Phi $=1.618033989$

2/Reference female $\mathrm{HGO}$ (rwHGO): rwHGO $=0.6913477936$

Error $(\mathrm{tHGO}-\mathrm{rwHGO})=0.6909830056-0.6913477936=$ $-0.0003647879784$

and

Reference male HGO (rmHGO): $\mathrm{rmHGO}=0.6922864236$

Error (tHGO - rmHGO) $=0.6909830056-0.6922864236=$ $-0.001303417973$

Details: HGOwoman $=\left[\left(\begin{array}{llll}\text { sum } & \mathrm{C}+\mathrm{G} & \text { single strand } 1 & \text { to } 22\end{array}\right.\right.$ chromosomes $)+($ sum $\mathrm{C}+\mathrm{G}$ chrX $)+($ sum $\mathrm{C}+\mathrm{G}$ single strand 1 to 22 chromosomes $)+($ sum $\mathrm{C}+\mathrm{G}$ chrX $)] /[$ (sum $\mathrm{T}+\mathrm{A}$ single strand 1 to 22 chromosomes $)+($ sum $\mathrm{T}+\mathrm{A}$ chrX $)+($ sum $\mathrm{T}+\mathrm{A}$ single strand 1 to 22 chromosomes $)+($ sum $\mathrm{T}+\mathrm{A}$ chrX)]

HGOman $=[$ (sum $\mathrm{C}+\mathrm{G}$ single strand 1 to 22 chromosomes $)+($ sum $\mathrm{C}+\mathrm{G}$ chrX $)+($ sum $\mathrm{C}+\mathrm{G}$ single strand 1 to 22 chromosomes $)+($ sum $\mathrm{C}+\mathrm{G}$ chrY) $] /[$ (sum $\mathrm{T}+\mathrm{A}$ single strand 1 to 22 chromosomes $)+($ sum $\mathrm{T}+\mathrm{A}$ chrX) + (sum $\mathrm{T}+\mathrm{A}$ single strand 1 to 22 chromosomes $)+($ sum $\mathrm{T}+\mathrm{A} \operatorname{chrY})]$

\section{Results and discussion}

In all that follows, the general methodology will be as follows: we calculate, for the 46 chromosomes constituting each genome studied, only the single-stranded DNA sequences. In these sequences, we count the relative populations of bases $\mathrm{T}+\mathrm{A}$ on the one hand, and $\mathrm{C}+\mathrm{G}$ on the other hand.

\section{I/ Genome unity}

HGO of the 3 whole genomes: Neanderthal, Sapiens Build34 and Sapiens HG38: The three genomes we compare here are differentiated on the one hand by their respective evolution levels, on the other hand by the sample of individual genomes of which they form the syntheses, and finally by the precision of the sequencing of DNA.

The detailed analysis related to the 3 whole genomes shows the various distances and errors between real computed HGOs for 
each genome and theoretical HGO optimum value $=0.6909830055$. Particularly, it is found that the 3 HGOs calculated for the respective 3 genomes of Neanderthal, Sapiens (2003 Build34 and $2013 \mathrm{hg} 38$ Sapiens) are very close to the ideal theoretical optimal $\mathrm{HGO}=0.6909830056(99.67 \%$ for the least optimal genome). It is also observed that female genomes (XX) are more optimal than male genomes (XY). On the other hand, the genomes of Neanderthal and Sapiens (Build34 of 2003) have very close optimization levels. We believe these results from the fact that the precisions of their respective DNA sequencing are similar. On the contrary, the hg 38 genomes of 2013 show the most optimal levels, this is most certainly due to the deeper quality of their DNA sequencing. Figure 1 summarises HGO results for these 3 human genomes of varying levels of evolution.

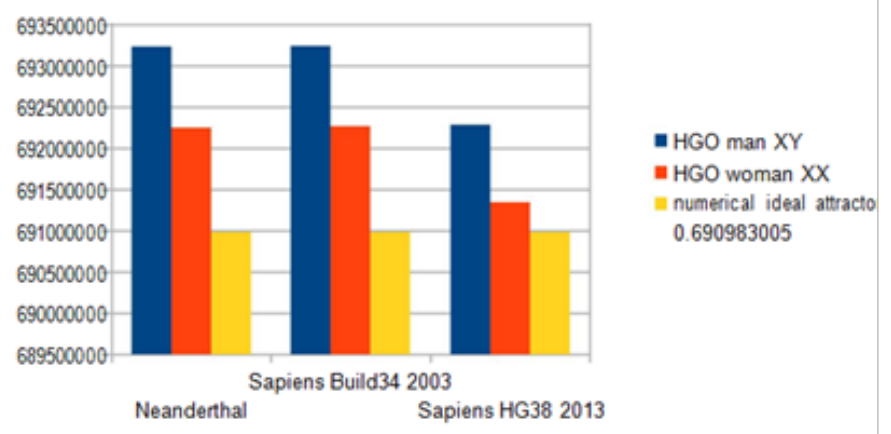

Figure I The respective HGOs of 3 human genomes of varying levels of evolution are shown here.

\section{Considerations on this theoretical Human Genetic Optimum (HGO) of (3-Phi)/2}

This formula is particularly simple. We can even make it more "beautiful", indeed:

Since $1+\mathrm{Phi}=\mathrm{Phi} * 2$, we can write:

$(3-\mathrm{Phi}) / 2=\mathrm{C}+\mathrm{G} / \mathrm{T}+\mathrm{A}=(4-(1+\mathrm{Phi})) / 2=(4-(\mathrm{Phi} * 2)) / 2=(2 * 2-$ $\left.\mathrm{Phi}^{*} 2\right) / 2=\mathrm{C}+\mathrm{G} / \mathrm{T}+\mathrm{A}$

This new equivalent formula contains only the numbers " 2 " and "Phi".

This omnipresence of the number " 2 " in this formula has a strong analogy with the predictive formula of the periodic table of the Mendeleiev elements, also built around the " 2 ". ${ }^{19}$

A second track to be studied could consist in replacing this writing by:

$(3-\mathrm{Phi}) / 2=(3-\mathrm{Phi}) /(5-3)=\mathrm{C}+\mathrm{G} / \mathrm{T}+\mathrm{A}$

By this artifice of writing, we thus make the " 3 " appear in the numerator and the denominator (!)

The formula then becomes:

$$
\begin{aligned}
& (3-P h i) \times(T+A)=2 \times(C+G)=(5-3) \times(C+G) \\
& 3(T+A)+3(C+G)=5(C+G)+P h i(T+A) \\
& 3(T+A+C+G)=5(C+G)+P h i(T+A)
\end{aligned}
$$

Therefore, if we consider that the single copy (single strand DNA) of the 24 chromosomes whole genomes XX or XY all lead to the same attractor $\mathrm{HGO}=(3-\mathrm{Phi}) / 2$, to write:
Considering the cumulative population of 24 chromosomes of the single human genome (single strand DNA)

We check the following perfect balance: "THREE times the whole genome $(\mathbf{T}+\mathbf{A}+\mathbf{C}+\mathbf{G})=$ FIVE times $(\mathrm{C}+\mathbf{G})$ PLUS Phi times $(\mathrm{T}+\mathrm{A})^{\prime \prime}$

Verification on 24 hg 38 chromosomes single strand DNA:

$\mathrm{CG}=1200551672$

$\mathrm{TA}=1737087441$

$3 \times(\mathrm{CG}+\mathrm{TA})=8812917339$

$(5 \times \mathrm{CG})+(\mathrm{PHI} \times \mathrm{TA})=8813424881$

$8812917339 \div 881342488=0.9999424126$

$8812917339-881342488={ }^{-} 507542$

Finally, it is remarkable that this formula is based on integers 3 or 5 . In fact, these numbers are very small integers and they are Fibonacci numbers. It will therefore be interesting to postpone the error calculations on the accuracy of these two integers 3 and 5:

\section{$(5 \times \mathrm{CG})+(\mathrm{Phi} \times \mathrm{TA})=8813424881 /(\mathrm{CG}+\mathrm{TA})=2937639113$ \\ $8813424881 / 2937639113=3.000172772$}

and

$3 \times(\mathrm{CG}+\mathrm{TA})=8812917339$

$(\mathrm{Phi} \times \mathrm{TA})=2810666521$

$8812917339-2810666521=6002250818$

$\mathrm{CG}=1200551672$

$6002250818 \div \mathrm{CG}=4.999577243$

The exact formula can then be written:

$3.000172772(\mathrm{~T}+\mathrm{A}+\mathrm{C}+\mathrm{G})=5(\mathrm{C}+\mathrm{G})+\mathrm{Phi}(\mathrm{T}+\mathrm{A})$

or

$3(\mathrm{~T}+\mathrm{A}+\mathrm{C}+\mathrm{G})=4.999577243(\mathrm{C}+\mathrm{G})+\mathrm{Phi}(\mathrm{T}+\mathrm{A})$

\section{2/Chromosomes hierarchy}

\section{HGO spectral hierarchy of the 24 human chromosomes}

The following 2 figures (Figure 2) and (Figure 3) illustrate the hierarchical spectrum of the individual HGOs of each of the 24 chromosomes for each of the three genomes analyzed. It should be noted that the upstream/ downstream tipping point lies between chromosomes 14 and 21, which is closely related to the probable mechanisms explaining trisomy 21 (whose disorders involve precisely these two chromosomes). Finally, we note that it is the downstream region (Figure 3) that contributes the most to the superiority of optimality of sapiens hg38 compared to sapiens Build34. We have sorted the 24 chromosomes by increasing values of CG/TA ratios in the 3 cases of compared genomes. It then reveals a hierarchical classification scale of 24 chromosomes ranging from 1/Phi (chromosome4) to 3/2 Phi (chromosome 19). 


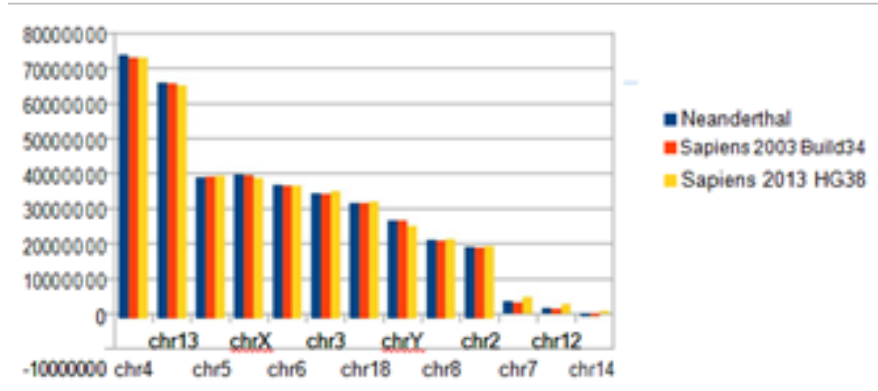

Figure 2 《UP » chromosomes: HGO diversity of human chromosomes UPSTREAM of the numerical attractor.

\section{Diversity of Chromosomes from 3 Human Genomes}

The Chromosomes DOWNSTREAM the HGO theoretical point $=0.6909630056$

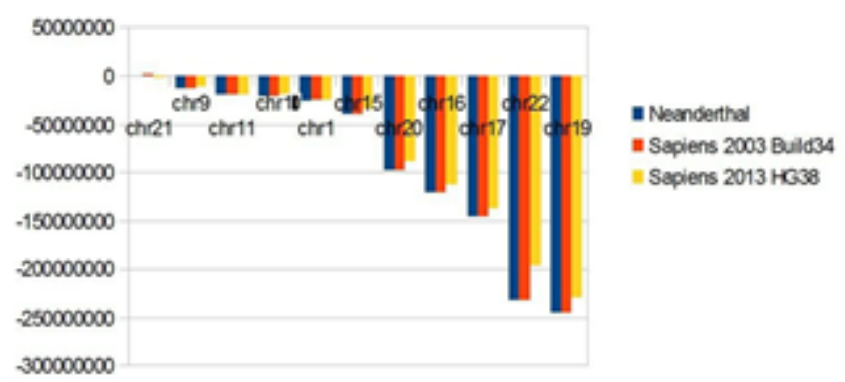

Figure 3 « Down » chromosomes: Diversity of HGOs of human chromosomes DOWNSTREAM of the numerical attractor $\mathrm{HGO}=0.6909830056$.

\section{3/Cohesion chromosomes/genome}

\section{About the hierarchical classification of 24 single stranded chromosomes}

In the following, we demonstrate a real interaction, a kind of "dialogue" with feddback between the equilibrium of the whole genome and the part of each of the individual chromosomes. We must now regulate this high level of remarkable numerical constraints which seem to "frame" the CG and TA populations of each of the 24 human chromosomes on one hand and of the entire genome on the other hand. This will be verified for the human HG38 reference genome, but - as illustrated in Table 2 below - these remarkable properties will be extended to other higher primates in. ${ }^{20}$ First, is there a simple relationship between $\mathrm{HGO}(\mathrm{P} 2)$, the numerical constraint at the scale of the entire genome, and the two extreme extremes of chromosome 4 (P1) and chromosome 19 (P3)? Then:

$$
\begin{aligned}
& \mathrm{P} 1=1 \div \mathrm{PHI}=0.6180339887 \\
& \mathrm{P} 3=3 \div(2 \times \mathrm{PHI})=0.927050983 \\
& \mathrm{P} 2=(3-\mathrm{PHI}) \div 2=0.6909830055
\end{aligned}
$$

We could compute:

P2-P1 $=0.07294901685$

\section{P3-P2 $=0.2360679775$}

Then, (P3-P2) $\div($ P2-P1) $=3.236067979$

Given that $2 \times \mathrm{PHI}=3.236067978$

Then, $(\mathrm{P} 3-\mathrm{P} 2) \div(\mathrm{P} 2-\mathrm{P} 1)=2 \times \mathrm{PHI}=3.236067979$

In other hand, P3-P1 $=1 \div(2 \times \mathrm{PHI})=0.3090169943$

Then finally, the high level of strong numerical constraints applied simultaneously to the 2 extrema chromosomes and to the whole human genome:

P1: chr4 1/Phi

$==>\mathrm{P} 2-\mathrm{P} 1=0.07294901685$

$\mathrm{P} 2$ : genome (3-Phi)/2

$==\mathrm{P} 3-\mathrm{P} 2=0.2360679775$

P3: chr19 3/2 Phi

Then:

$(\mathrm{P} 3-\mathrm{P} 2) \div(\mathrm{P} 2-\mathrm{P} 1) 3.236067979=2 \times \mathrm{PHI}=3.236067978$

P3-P1 $0.30901699431 \div(2 \times$ PHI $) 0.3090169943$

\section{4/Closure}

We will now demonstrate a very strong property of the human genome very close to the theory of the autopoiesis of my friend franco-chilian biologist Francisco Varela. ${ }^{21,22}$ In this theory, the coherence, consistency and integrity of living systems are modeled: the DNA of the human genome is a wonderful illustration of this. Let us now look at the two UP chromosome populations (chr4 to chr14) and DOWN (chr21 to chr19). Would there exist particular constraints or remarkable relations on these 2 populations of chromosomes which determine the law described here? Let us recall in (Table 1) the respective populations and ratios of each of the 24 chromosomes of the genome HG38:

Then cumulating in Table1 he populations $\mathrm{C}+\mathrm{G}$ and $\mathrm{T}+\mathrm{A}$ in each subclass UP and DOWN:

$\mathrm{UP}=7423983031124171661$

DOWN=458153369 612915780

DOWN/UP: $\mathrm{C}+\mathrm{G}$ T $+\mathrm{A}$

0.61712609950 .5452154695

or UP / DOWN : $\mathrm{C}+\mathrm{G}$ T $+\mathrm{A}$

$$
1.620414371 .834137246
$$

This result is remarkable since it means that: on the one hand, the CG/TA ratio of chromosome4, a sort of leader or "semaphore", is equal to $1 / \mathrm{Phi}$. On the other hand, the ratio of the $\mathrm{C}+\mathrm{G}$ ratios between the 11 DOWN chromosomes to the 13 UP chromosomes is also equal to $1 /$ Phi.

Table I Evidence of strong numerical constraints surrounding the relative populations $C+G / T+A$ constituting the hierarchical meta structure of the 24 chromosomes in humans and large primates

\begin{tabular}{lllllll}
\hline Genome & \multicolumn{2}{l}{ Extremum top CG/TA chr4 } & \multicolumn{2}{l}{ Extremum down CG/TA chr19 } & Spectral limits (CG/TA chr19)-(CG/TA chr4) \\
\hline & value & $\begin{array}{l}\text { Error CG/TA Chr4 } \\
\text { vs I/Phi }\end{array}$ & value & $\begin{array}{l}\text { Error CG/TA chr19 } \\
\text { vs 3/2 Phi }\end{array}$ & value & \multicolumn{1}{c}{$\begin{array}{l}\text { Error ( 3/2 Phi ) - Spectral } \\
\text { Limits }\end{array}$} \\
\hline Sapiens HG38 & 0.619261918 & -0.0012279291 & 0.920811 & 0.006240148 & 0.301549 & 0.007468078 \\
\hline
\end{tabular}


Table Continued....

\begin{tabular}{|c|c|c|c|c|c|c|}
\hline \multirow[t]{2}{*}{ Genome } & \multicolumn{2}{|c|}{ Extremum top CG/TA chr4 } & \multicolumn{2}{|c|}{ Extremum down CG/TA chrI9 } & \multicolumn{2}{|c|}{ Spectral limits (CG/TA chr I 9)-(CG/TA chr4) } \\
\hline & value & $\begin{array}{l}\text { Error CG/TA Chr4 } \\
\text { vs I/Phi }\end{array}$ & value & $\begin{array}{l}\text { Error CG/TA chrl9 } \\
\text { vs } 3 / 2 \mathrm{Phi}\end{array}$ & value & $\begin{array}{l}\text { Error ( } 3 / 2 \text { Phi ) - Spectral } \\
\text { Limits }\end{array}$ \\
\hline Sapiens BUILD34 & 0.619377817 & -0.001343828 & 0.936495 & $-0.009444 I 77$ & 0.317117 & -0.00810035 \\
\hline neanderthal & 0.618590097 & -0.000556108 & 0.936648 & -0.009596744 & 0.318058 & -0.009040636 \\
\hline chimp & 0.615238866 & $0.002795 \mid 23$ & 0.92794 & -0.000888599 & 0.312701 & -0.003683723 \\
\hline Orangutang & 0.614364584 & 0.003669404 & 0.925221 & 0.001829533 & 0.310857 & $-0.00|83987|$ \\
\hline gorilla & 0.617745603 & 0.000288386 & 0.929942 & -0.002890887 & 0.312196 & -0.003179272 \\
\hline macaque & 0.653660819 & $-0.03562683 \mid$ & 0.929994 & -0.002942726 & 0.276333 & 0.032684105 \\
\hline
\end{tabular}

Table 2 Respective populations and ratios of each of the 24 chromosomes of human genome HG38 (2013)

\begin{tabular}{|c|c|c|c|}
\hline Chromosome & $\mathbf{C}+\mathbf{G}$ & $\mathbf{T}+\mathbf{A}$ & $(\mathbf{C}+\mathbf{G}) /(\mathrm{T}+$ \\
\hline \multicolumn{4}{|c|}{ Chromosomes UPSTREAM HGO point $=(3-\mathrm{Phi}) \div 2=0.690983005$} \\
\hline 4 & $7256800 \mathrm{I}$ & 117184666 & 0.619261918 \\
\hline 13 & 37772797 & 60210328 & $0.62734747 \mid$ \\
\hline 5 & 71611274 & 109654104 & $0.653065|5|$ \\
\hline$x$ & 61221521 & 93671508 & 0.653576763 \\
\hline 6 & 67360020 & 102718502 & 0.655772998 \\
\hline 3 & 78577742 & | | 9522393 & 0.65743|| $3 \mid$ \\
\hline 18 & 31856106 & 48233499 & 0.660456045 \\
\hline Y & 10572683 & 15842360 & 0.66736793 \\
\hline 8 & 58133960 & 86634176 & 0.671028025 \\
\hline 2 & 96769083 & $|43779| 45$ & 0.673039772 \\
\hline 7 & 64696843 & 94273288 & 0.686269084 \\
\hline 12 & 54275482 & 78862334 & 0.688230734 \\
\hline 14 & 36982791 & 53585358 & 0.690165978 \\
\hline
\end{tabular}

Chromosomes DOWNSTREAM HGO point $=(3-\mathrm{Phi}) \div 2=0.6909830056$

\begin{tabular}{llll}
21 & 16411625 & 23676994 & 0.693146478 \\
9 & 50270473 & 71520077 & 0.70288617 \\
11 & 55885058 & 78648684 & $0.7105657 \mid$ \\
10 & 55359481 & $7790348 \mid$ & 0.710616269 \\
1 & $9616657 \mid$ & $13431444 \mid$ & 0.715980875 \\
15 & 35578844 & $4906248 \mid$ & $0.72517417 \mid$ \\
20 & 28010605 & 35933652 & 0.779508996 \\
16 & 36472718 & 45333225 & $0.804547 \mid 73$ \\
17 & 37575444 & 45344760 & 0.828661217 \\
22 & 18406838 & 20752939 & 0.886950904 \\
19 & 28015712 & 30425046 & 0.920810835 \\
\hline
\end{tabular}

\section{Closure varela's theory}

Distance amplitudes. CG/TA Down/Up=(P3-P2)/(P2-P1)=2 Phi

Distance populations CG Down/Up=CG Down/CG Up =1/Phi
Then, distance amplitudes Up/Down CG/TA=1/2Phi

Populations CG Down/CG Up=2 times Distance amplitudes CG/ TA Up/Down.

It is remarkable to obtain this relation between AMPLITUDES on the one hand, and POPULATIONS $(\mathrm{C}+\mathrm{G})$ on the other hand. We thus find again this number " 2 ", symbol of the doubling of frequency such as the octave shift in music ... suggesting the possible wave nature of the DNA. ${ }^{23}$ We still have a lot to discover on this fascinating CODE that is DNA. ${ }^{24-32}$

\section{Conclusion}

In order to convince the most skeptical about this NUMERICAL UNIT of the human genome, it seemed judicious to use the analogy: By taking the notation invented by the mathematician Leibnitz, we will imagine a population of 3.5 billion "monads", basic TCAG nucleotides, constituting the single strand of DNA of our genome. In the course of human evolution, these Monads self-organized themselves into 23 clusters, the pairs of chromosomes. A first source of astonishment will be the finely adjusted "classification" of the 24 chromosomes: Indeed, the ratios of their respective populations $(\mathrm{C}+\mathrm{G}) /(\mathrm{T}+\mathrm{A})$ are not arbitrary, they adjust according to a kind of "Musical range" between 1/Phi (P1: chromosome 4) and 3/2 Phi (P3: chromosome 19). Let an amplitude of variation (P3-P1) equal to $1 / 2$ Phi. Now this ratio 2 corresponds in the world of waves to a doubling of frequency while in the musical universe it corresponds to an octave. In addition, attenuation (here between $\mathrm{P} 1$ and $\mathrm{P} 3$ ) or variation factors of $1.5(50 \%)$ are commonly used in acoustics, electronics and more generally in the physical sciences. A second source of astonishing will be this kind of "center of gravity", a kind of "average" constituted by this general ratio at the scale of the whole genome: $\mathrm{P} 2:(\mathrm{C}+\mathrm{G}) /$ $(\mathrm{T}+\mathrm{A})=(3-\mathrm{Phi}) / 2=\left(4-\left(\mathrm{Phi}^{*} 2\right)\right) / 2=(2 * 2-\mathrm{Phi} * 2) / 2$. The reader will notice both the simplicity and the "beauty" of this formula constructed around the number " 2 " $(2 * 2-\mathrm{Phi} * 2) / 2$.

However, a third source of astonishment will come from the link, the numerical relationship uniting the 2 levels of organizations above: AUTONOMOUS organizations and DIVERSITY of each of the 24 chromosomes on the one hand and global organization and UNITY of the whole genome in other hand. How can one be surprised at this real COHESION between the diversified and perfectly "bounded" hierarchy of the 24 chromosomes and the unity of the entire genome? In fact, if (P3-P2) represents the chromosomes beyond the equilibrium point of the genome (3-Phi)/2 while (P2-P1) represents the chromosomes before the equilibrium point of the genome, then (P3$\mathrm{P} 2) /(\mathrm{P} 2-\mathrm{P} 1)=2 \times \mathrm{Phi}$, whereas $\mathrm{P} 3-\mathrm{P} 1=1 /(2 \times \mathrm{Phi})$. Again this famous "2" report uniting the cluster scales of individual chromosomes and 
the entire genome scale. Finally, a fourth and last source of astonishing will be this evidence of a "closure" in the sense of the autopoiesis theory of Francisco Varela and Humberto Maturana: While, as a kind of "guide" chromosome, the chromosome4 "resonates" in a ratio $(\mathrm{C}+\mathrm{G}) /(\mathrm{T}+\mathrm{A})=1 / \mathrm{Phi}$, on the one hand, the same "mirror resonance" in echo is discovered between the ratio of the populations $(\mathrm{C}+\mathrm{G})$ of the chromosomes "down" located after the genomic equilibrium point, and the population $(\mathrm{C}+\mathrm{G})$ of the set of « up » chromosomes before the genomic equilibrium point, here also $1 / \mathrm{Phi}$. We can indeed speak of global cohesion of the genome because of this double constraint on the boundaries of the extreme chromosomes (P1: chr4 and P3: chr19) on the one hand, and on the contents of the $\mathrm{C}+\mathrm{G}$ ratios of all other chromosomes Intermediaries on the other.

We can only conclude this "cohesion", this consistency of the human genome in the sense of the closing in a "feedback" or "closure" demonstrating that the human genome behaves like an "ALL", in harmony with each of its 24 chromosomes. Finally, our approach may be related to these hundreds of unpredictable mutations resulting from manipulation of genomes by CRISPR revolutionary technology. ${ }^{33-35}$ Effectively in their 2017 article, authors note that « .../...They found that the technique had successfully corrected a gene that causes blindness in the mice, but the two mice that had undergone CRISPR gene-editing had sustained more than 1,500 unintended single-nucleotide mutations, and more than 100 larger deletions and insertions.

\section{Acknowledgements}

We especially thank Dr. Robert Friedman (MD. practiced nutritional and preventive medicine in Santa Fe, New Mexico), worlwide expert on Golden ratio applications. We also thank the mathematician Pr. Diego Lucio Rapoport (Buenos aires), the french biologist Pr. François Gros (Pasteur institute,co-discoverer of RNA messenger with James Watson and Walter Gilbert ) and Pr. Luc Montagnier, medicine Nobel prizewinner for their interest in my research of biomathematical laws of genomes.

\section{Conflict of interest}

The author declares no conflict of interest.

\section{References}

1. Suzuki K, Tsunekawa Y, Hernandez-Benitez R, et al. In vivo genome editing via CRISPR/Cas 9 mediated homology-independent targeted integration. Nature. 2016;540(7631):144-149.

2. Lieberman-Aiden, Van Berkum NL, Williams L, et al. Comprehensive mapping of long-range interactions reveals folding principles of the human genome. Science. 2009;326(5950):289-293.

3. Perez JC. Chaos DNA and neuo-computers: a golden link. Speculations in Science and Technology. 1991;14:336-346.

4. Marcer PJ. Order and chaos in DNA-the denis guichard prizewinner: jean-claude Perez. Kybernetes. 1992;21(2):60-61.

5. Perez JC. L'adn Décrypté. Belgium: Resurgence publisher Liege; 1997.

6. Perez JC. Codex Biogenesis. Resurgence, Belgium: 2009.

7. Perez JC. Sapiens Mitochondrial DNA Genome Circular long range numerical meta structures are highly correlated with cancers and genetic diseases mtDNA Mutations. J Cancer Sci Ther. 2017;9:512-527.

8. Perez JC. DUF1220 Homo Sapiens and Neanderthal fractal periods architectures breakthrough. SDRP Journal of Cellular and Molecular Physiology. 2017;1(1):1-37.
9. Perez JC. Global and long range fractal differences between sapiens and neanderthal genomes. To be published in 2017; 2017.

10. Perez JC. Codon Populations in single-stranded whole human genome DNA are fractal and fine-tuned by the golden ratio 1.618 . interdisciplinary sciences: Computational Life Sciences. 2010;2(3):228-240.

11. Perez JC. Caminos Interdisciplinaios. Seminario CLAVE_INTER, Espacio Interdisciplinario, Uruguay: Universidad de la Republica Montevideo; 2011. p. 1-27.

12. Perez JC. Decoding non-coding DNA codes: human genome meta-chromosomes architecture. BIT Life Sciences' 3rd Annual world vaccine congress; Beijing, China: 2011.

13. Perez JC. The 3 Genomic numbers discovery: how our genome single-stranded DNA sequence is self-designed as a numerical whole. Applied Mathematics. 2013;4(1):37-53.

14. Perez J. Deciphering hidden DNA meta-codes. The great unification \& master code of biology. J Glycomics Lipidomics. 2015;5:131.

15. Perez JC. Fractal Self-similarity, Scale Invariance and Stationary waves (to be published 2017); 2017.

16. Kay Prüfer, Fernando Racimo, Nick Patterson, et al. The complete genome sequence of a Neanderthal from the Altai Mountains. Nature. 2014;43-49.

17. Sapiens Build. Finishing the euchromatic sequence of the human genome. Nature. 2004;431:931-945.

18. Sapiens HG38. Human genome overview; 2013.

19. perez JC. Symmetry and asymmetry in the MENDELEEV's periodic table predictive EQUATION. SDRP Journal of Computational Chemistry \& Molecular Modelling. 2017;2(1):1-5.

20. Perez JC. Humans and Primates Chromosomes4 Fractal CODES: periodic stationnary waveforms charaterizing and differenciating Neanderthal and Sapiens whole chromosomes DNA sequences; 2017.

21. Varela FJ. Autonomy and autopoiesis. In: Roth G, et al. editors. Selforganizing systems: An interdiciplinary approach. Frankfurt/New York, USA: Campus Verlag; 1981. p.14-24.

22. Maturana HR, Varela FJ. The cognitive process. Autopoiesis and cognition: the realization of the living, Netherlands: Springer; 1980. p. 1-3.

23. Montagnier L, Aïssa J, Capolupo A, et al. Water bridging dynamics of polymerase chain reaction in the gauge theory paradigm of quantum fields. Water. 2017;9(5):339.

24. Friedman R, Cross M. The golden ratio \& fibonacci sequence: golden keys to your genius, health, wealth \& excellence 1st ed. USA: Hoshin Media; 2013.

25. Pellionisz AJ, Graham R, Pellionisz PA, et al. Recursive Genome Function of the Cerebellum: Geometric Unification of Neuroscience and Genomics. In: Manto M, DL, et al. Editors. Handbook of the Cerebellum and Cerebellar Disorders. 1st ed. Netherlands: Springer; 2006. p. 13811423 .

26. Rapoport DL. Möbius strip and Klein bottle genomic topologies, self reference, harmonics and evolution. Quantum Biosystem. 2015;7(1):107174.

27. Persaud, Dharam, Leary O, James P. Fibonacci series, golden proportions, and the human biology. HWCOM Faculty Publications. 2015;7(2):1-7.

28. Roman V, Yampolskiy. On the origin of synthetic life: attribution of output to a particular algorithm. Physica Scripta. 2017;99(2):1-16.

29. The Royal Swedish Academy of Sciences Phys Scr ; 20117.

30. Perez JC. Decyphering the MASTER CODE® structure and discovery of a periodic invariant unifying $160 \mathrm{HIV} 1 / \mathrm{HIV} 2 / \mathrm{SIV}$ isolates genomes. Biomed J Sci \& Tech Res. 2017;1(2):1-3. 
31. Perez JC. The master code of DNA: towards the discovery of the SNPs function (single-nucleotide polymorphism). J Clin Epigenet. 2017;3(3):1-9.

32. Perez JC. The Master Code of Biology: Self-assembly of two identica Peptides beta A4 1-43 Amyloid in Alzheimer's Diseases. Biomed J Sci \& Tech Res. 2017;1(4):1-5.

33. Perez JC. The Master Code of Biology: from prions and prionslike invariants to the self-assembly thesis. Biomed $J$ Sci \& Tech Res. 2017;1(4):1-3
34. Kellie A Schaefer, Wen-Hsuan Wu, Diana F Colgan, et al. Unexpected mutations after CRISPR-Cas9 editing in vivo. Nature. 2017;14:547-548.

35. Perez JC. Predicting LOH Deletions pathogenicity (loss of heterozygosity) in breast, colorectal, prostate, glioblastoma, and neuroblastoma cancers. Global Journal of Medical Research. 2017;17(6):1. 